\title{
Separate groups of dopamine neurons innervate caudate head and tail encoding flexible and stable value memories
}

\section{Hyoung F. Kim*, Ali Ghazizadeh and Okihide Hikosaka}

Laboratory of Sensorimotor Research, National Eye Institute - National Institutes of Health, Bethesda, MD, USA

\section{Edited by:}

Jose L. Lanciego, University of

Navarra, Spain

Reviewed by:

Yoland Smith, Emory University, USA Gordon William Arbuthnott, Okinawa Institute of Science and Technology, Japan

Satoshi Ikemoto, National Institute on Drug Abuse - National Institutes of Health, USA

\section{*Correspondence:}

Hyoung F. Kim, Laboratory of Sensorimotor Research, National Eye Institute - National Institutes of Health, Bethesda, MD 20892, USA e-mail: hyoung.f.kim@gmail.com
Dopamine (DA) neurons are thought to be critical for reward value-based learning by modifying synaptic transmissions in the striatum. Yet, different regions of the striatum seem to guide different kinds of learning. Do DA neurons contribute to the regional differences of the striatum in learning? As a first step to answer this question, we examined whether the head and tail of the caudate nucleus of the monkey (Macaca mulatta) receive inputs from the same or different DA neurons. We chose these caudate regions because we previously showed that caudate head neurons learn values of visual objects quickly and flexibly, whereas caudate tail neurons learn object values slowly but retain them stably. Here we confirmed the functional difference by recording single neuronal activity while the monkey performed the flexible and stable value tasks, and then injected retrograde tracers in the functional domains of caudate head and tail. The projecting dopaminergic neurons were identified using tyrosine hydroxylase immunohistochemistry. We found that two groups of DA neurons in the substantia nigra pars compacta project largely separately to the caudate head and tail. These groups of DA neurons were mostly separated topographically: head-projecting neurons were located in the rostral-ventral-medial region, while tailprojecting neurons were located in the caudal-dorsal-lateral regions of the substantia nigra. Furthermore, they showed different morphological features: tail-projecting neurons were larger and less circular than head-projecting neurons. Our data raise the possibility that different groups of DA neurons selectively guide learning of flexible (short-term) and stable (long-term) memories of object values.

Keywords: nigrostriatal pathway, dopamine neuron, substantia nigra pars compacta, parallel circuit, object value learning, macaque monkey

\section{INTRODUCTION}

The basal ganglia are thought to be essential for the selection of action (Mink, 1996). Particularly relevant to this function is the striatum whose outputs could be used to facilitate a desired action (through the direct pathway) and inhibit undesired actions (through the indirect pathway) (Hikosaka etal., 2000; Frank etal., 2004; McHaffie etal., 2005). Notably, the striatum is a large structure which receives cognitive/sensorimotor/emotional signals from cortical areas (including association, sensorimotor, and limbic cortices) and subcortical areas [including thalamus and substantia nigra (SN)] (Selemon and Goldman-Rakic, 1985; Saint-Cyr et al., 1990; Parent and Hazrati, 1995; McHaffie etal., 2005). Hence, the basal ganglia are composed of multiple circuits for different kinds of action selection (Alexander etal., 1986). Such multiple mechanisms are likely to be deployed during learning of actions leading to rewarding outcomes. It has been suggested that two different regions of the striatum contribute to action learning differently (Hikosaka etal., 1999): the association striatum mainly in the early stage of learning; the sensorimotor striatum mainly in the late stage of learning. The association and sensorimotor striatum may correspond to the dorsomedial and dorsolateral striatum in rodents (Yin and Knowlton, 2006), and the rostral caudate/putamen and caudal putamen in monkeys (Miyachi et al.,
1997, 2002) and humans (Lehéricy etal., 2005; Seger etal., 2010).

Our recent studies have shown that the caudate nucleus (CD) of the monkey also contains different learning mechanisms for choosing good objects. Neurons in the head of the caudate nucleus (CDh) change their responses to visual objects quickly depending on the reward values recently associated with the objects (flexible values), whereas neurons in the tail of the caudate nucleus (CDt) change their responses slowly depending on the reward values consistently associated with the objects (stable values; Kim and Hikosaka, 2013; Yamamoto et al., 2013). Both the flexible and stable value signals are sent to the superior colliculus via the SN pars reticulata (Hikosaka et al., 2014) and used to orient the monkey's gaze to good (high-valued) objects (Kim and Hikosaka, 2013).

How does the object value learning occur in $\mathrm{CDh}$ and $\mathrm{CDt}$ ? A key factor may be the input from dopamine (DA) neurons which carry reward-related signals (Schultz, 1998), heavily innervate the entire striatum (Richfield et al., 1987; Kato et al., 1995), and regulate synaptic plasticity in striatal neurons (Reynolds and Wickens, 2002). However, it is unknown how the object value learning occurs at different speeds in $\mathrm{CDh}$ and $\mathrm{CDt}$. We considered two possible mechanisms. First, the difference may be caused by different learning mechanisms between $\mathrm{CDh}$ and 
CDt neurons, while they receive DA inputs non-selectively. Second, the difference may be caused by selective inputs from DA neurons carrying different learning signals. As a first step to test these hypotheses, we examined anatomically whether the fastlearning $\mathrm{CDh}$ and the slow-learning $\mathrm{CDt}$ receive inputs from the same or different DA neurons. Our data indicate that two spatially separate groups of DA neurons innervate $\mathrm{CDh}$ and CDt. Furthermore, these groups of DA neurons have different morphologies.

\section{MATERIALS AND METHODS GENERAL PROCEDURES}

Three adult male rhesus monkeys (Macaca mulatta, 8-10 kg; monkey SM and ZO for neuronal recording and tracer study, and DW for neuronal recording) were used for the experiments. Monkeys DW and ZO were used in our previous study (Kim and Hikosaka, 2013), but we added more neuronal data in this study. All animal care and experimental procedures were approved by the National Eye Institute Animal Care and Use Committee and complied with the Public Health Service Policy on the humane care and use of laboratory animals. We implanted a plastic head holder and a recording chamber to the skull under general anesthesia and sterile surgical conditions. The chamber was tilted laterally by $25^{\circ}$ and was aimed at $\mathrm{CDh}$ and CDt. Two search coils were surgically implanted under the conjunctiva of the eyes to record eye movements. After the monkeys fully recovered from surgery, we started training them with short-term flexible and long-term stable value procedures.

\section{NEURONAL RECORDING}

While the monkey was performing a task, we recorded the activity of single neurons in different subregions of CD using conventional methods. The recording sites were determined with $1 \mathrm{~mm}$ spacing grid system, with the aid of MR images (4.7T, Bruker) obtained along the direction of the recording chamber. Single-unit recording was performed using glass-coated electrodes (AlphaOmega). The electrode was inserted into the brain through a stainless-steel guide tube and advanced by an oil-driven micromanipulator (MO-97A, Narishige). The electric signal from the electrode was amplified with a band-pass filter $(200-10 \mathrm{kHz}$; BAK). Neural spikes were isolated online using a custom voltage-time window discrimination software (MEX, Laboratory of Sensorimotor Research, National Eye Institute - National Institutes of Health [LSR/NEI/NIH]) and their timings were detected at $1 \mathrm{kHz}$. The waveforms of individual spikes were collected at $50 \mathrm{kHz}$.

\section{BEHAVIORAL TASKS}

Behavioral tasks were controlled by a QNX-based real-time experimentation data acquisition system (REX, LSR/NEI/NIH). The monkey sat in a primate chair, facing a frontoparallel screen in a sound-attenuated and electrically shielded room. Visual stimuli generated by an active matrix liquid crystal display projector (PJ550, ViewSonic) were rear projected on the screen. We created the visual stimuli using fractal geometry. Their sizes were $\sim 8^{\circ} \times 8^{\circ}$.

\section{SHORT-TERM FLEXIBLE AND LONG-TERM STABLE BEHAVIORAL PROCEDURES}

To find functional domains in $\mathrm{CD}$, we used two behavioral procedures as previously described (Kim and Hikosaka, 2013).

\section{Short-term flexible value procedure}

This procedure allowed us to examine neuronal encoding of shortterm flexible object values as they were being updated in blocks of trials. Therefore, learning (of object values) and testing (of the neuronal activity) were done in one task procedure. For each monkey, a fixed set of two fractal objects (say, A and B) was used as the saccade target. Each trial started with a central white dot presentation, which the monkey was required to fixate. After $700 \mathrm{~ms}$, while the monkey was fixating on the central spot, A or B was chosen pseudo-randomly and was presented at the neuron's preferred position or at the diagonally symmetric position. Before this procedure, we determined the neuron's preferred position using a saccade task in which another fractal, as the target, was presented at multiple positions. During the flexible value procedure, the fixation spot disappeared $400 \mathrm{~ms}$ later, and then the monkey was required to make a saccade to the object within $4 \mathrm{~s}$. The monkey received a liquid reward $300 \mathrm{~ms}$ after making a saccade to one object (e.g., A) but received no reward after making a saccade to the other object (e.g., B). During a block of 3040 trials, the object-reward contingency was fixed, but it was reversed in a following block (e.g., B-high/A-low) without any external cue.

\section{Long-term stable value procedure}

To examine neuronal encoding of long-term stable object values, we conducted the learning procedure and the testing procedure separately on different days. In the learning procedure, the monkey experienced visual objects repeatedly in association with consistent reward values and thus learned their stable values. In the testing procedure, monkey's saccade behavior and neuronal activity were examined using different tasks. To focus on stable object values, we applied the testing procedure to objects that had been learned for more than four daily sessions. Below we explain in detail (1) the learning procedure, and (2) the procedure for testing neuronal activity.

Procedure for learning long-term stable object values. To create a fixed bias among fractal objects in their reward values, we used an object-directed saccade task. In each session, a set of eight fractals was used as the target and was presented at one of five positions (right, up, left, bottom, and center). The monkey made a saccade to the target to obtain a liquid reward. Half of the fractals were always associated with a liquid reward (highvalued objects), whereas the other half were associated with no reward (low-valued objects). One training session consisted of 160 trials (20 trials for each object). Each set was learned in one learning session in 1 day. The same sets of fractals were used repeatedly for learning across days (or months), throughout which each object remained to be either a high-valued object or a low-valued object. By the end of this study, monkeys SM, $\mathrm{ZO}$, and DW learned the stable values of 272, 456, 608 objects, respectively. 
Procedure for testing neuronal activity. To test the neuronal coding of stable object values, we used a passive-viewing task. In each session, a set of eight fractal objects was used as the visual stimuli. While the monkey was fixating on a central spot of light, some of the fractal objects ( $n=2-6)$ were chosen pseudo-randomly and presented sequentially in the neuron's preferred position in a pseudorandom order (presentation time: $400 \mathrm{~ms}$, inter-object interval time: $500-700 \mathrm{~ms}$ ). The preferred position was determined using the passive-viewing task in which another fractal was presented at various positions. After every one to four object presentations, a reward was delivered $300 \mathrm{~ms}$ later. The reward was thus not associated with particular objects. Each object was presented at least six times in one session. The neuronal coding of stable object values was tested after long-term learning (more than four daily sessions) and after a sufficient retention period ( $>1$ day after the last learning session). For each neuron, we used multiple sets of well-learned objects (2-4 sets, or 16-32 objects) to test its stable value-coding.

\section{NEURONAL VALUE-CODING}

We analyzed the neuronal discriminations of high-valued and lowvalued objects as previously described (Kim and Hikosaka, 2013). The statistical significance of the value-coding was determined for each neuron using ranksum test which compared the neuron's responses (i.e., number of spikes during the object presentation) to high- and low-valued objects across trials. To illustrate the distribution of value-coding neurons in $\mathrm{CD}$, we divided $\mathrm{CD}$ into $1 \mathrm{~mm} \times 1 \mathrm{~mm}$ voxels (projected to the sagittal plane) and calculated the proportion of value-coding neurons (number of value-coding neurons divided by all object-responsive neurons) in each voxel.

To show the overall value-coding in $\mathrm{CDh}$ and $\mathrm{CDt}$, we used the preferred and non-preferred responses to object values, since some $\mathrm{CD}$ neurons responded more strongly to high-valued objects (i.e., positive neurons) while others to low-valued objects (i.e., negative neurons). We first determined each neuron's preferred value by comparing the magnitude of the neuron's response to high-valued objects and to low-valued objects. This was done by computing a receiver operating characteristic (ROC) area based on the numbers of spikes within the test window in individual trials. We then averaged the responses of individual neurons in each subregion separately for the neurons' preferred value and the non-preferred value. This was done by using a crossvalidation method. Specifically, trials in one recording session were divided into the odd and even numbered trials. Either odd or even numbered trials were randomly chosen for determining the neuron's preferred value (using the ROC analysis), and the other was used for computing the average response. The cross-validation method precluded any artificial result of neuronal discrimination due to an arbitrary choice of the preferred value.

\section{RETROGRADE TRACER INJECTION}

To decide the injection sites, we recorded from single neurons throughout CD (except for CD genu) in monkey SM, ZO and DW, and examined if they encoded flexible or stable object values. Based on the value-coding map, we chose three injection sites in monkey
$\mathrm{SM}$ and ZO: one in $\mathrm{CDh}$ and two in $\mathrm{CDt}$. As retrograde tracers, we used Diamidino Yellow (DY; Sigma), Fast Blue (FB; Polyscience), and cholera toxin subunit B conjugated with Alexa 488 or Alexa 555 (CTB488 and CTB555; Life technologies). For FB, CTB488 and CTB555 injections, we used a custom-made injectrode consisting of an epoxy-coated tungsten microelectrode (FHC) for neuron recording and a silica tube (outer/inner tip diameter: 155/75 $\mu \mathrm{m}$; Polymicro technologies) for tracer injection. For DY injection, we used a 30-gage stainless-steel needle. A 10- $\mu \mathrm{L}$ Hamilton syringe held in a manual infusion pump (Stoelting) was used to inject $0.3 \mu \mathrm{l} \mathrm{FB} \mathrm{(3 \%} \mathrm{in} \mathrm{distilled} \mathrm{water),} 0.3 \mu \mathrm{l}$ CTB488, and $0.3 \mu \mathrm{l}$ CTB555 ( $1 \%$ in $0.01 \mathrm{M}, \mathrm{pH} 7.4$, phosphate buffer) at a speed of $0.01 \mu \mathrm{l} / \mathrm{min}$ and $1 \mu \mathrm{l}$ or $0.6 \mu \mathrm{l}$ DY $(2 \%$ in $0.2 \mathrm{M}, \mathrm{pH} 7.2$, phosphate buffer) at a speed of $0.02 \mu \mathrm{l} / \mathrm{min}$. After the injection, the injectrode or injection needle was left for $1 \mathrm{~h}$ to minimize tracer diffusion along the injectrode/needle track. For monkey SM, each of DY, FB, and CTB555 were injected at two adjacent sites in CDh and CDt: 3 and $4 \mathrm{~mm}$ anterior to the anterior commissure (AC) in $\mathrm{CDh}$ (for DY); 8 and $9 \mathrm{~mm}$ (for $\mathrm{FB}$ ) and 13 and $14 \mathrm{~mm}$ (for CTB555) posterior to AC in CDt. For monkey ZO, DY, CTB488, and CTB555 were injected at $3 \mathrm{~mm}$ anterior, $8 \mathrm{~mm}$ posterior, and $14 \mathrm{~mm}$ posterior to $\mathrm{AC}$, respectively.

\section{HISTOLOGY}

Two weeks after the tracer injection, monkey SM and ZO were deeply anesthetized with an overdose of sodium pentobarbital and perfused transcardially with saline followed by $4 \%$ paraformaldehyde. The head was fixed to the stereotaxic frame, and the brain was cut into blocks in the coronal plane including midbrain region. The block was post-fixed overnight at $4 \mathrm{C}^{\circ}$, and then cryoprotected for 5 days in increasing gradients of glycerol solution (5, 10 to $20 \%$ glyceorol in PBS) before being frozen. Frozen block was cut every $50 \mu \mathrm{m}$ using a microtome. Every $250 \mu \mathrm{m}$-interval slices were used for cell counting, and the adjacent two slices were used for Nissl and $\mathrm{TH}$ (tyrosine hydroxylase) staining. To examine the proportion of CTB- or DY-labeled neurons among all TH-positive neurons, we counted the $\mathrm{TH}$-positive neurons in three representative slices in the rostral, middle, and caudal regions of SNc in each monkey and calculated the proportion.

\section{IMMUNOCYTOCHEMISTRY}

To test if the CDt-projecting neurons were dopaminergic, we double-labeled $500 \mu \mathrm{m}$-interval SN slices with CTB and TH antibodies. After $1 \mathrm{~h}$ permeabilization with $0.05 \%$ Triton X-100 in TBS, the slices were blocked for $1 \mathrm{~h}$ in a solution containing $3 \%$ normal goat serum, $2 \%$ bovine serum albumin, and $0.05 \%$ Triton $\mathrm{X}-100$ in TBS and then incubated with rabbit anti-CTB (1:1500; GenWay) and mouse anti-TH (1:3000; Immunostar) antibody overnight at room temperature. After three washes with PBS, the slices were incubated for $2 \mathrm{~h}$ at room temperature with goat antimouse IgG antibody conjugated with Alexa 488 for monkey SM or Alexa 647 for monkey ZO (1:200; Invitrogen) and goat anti-rabbit IgG conjugated with Alexa 594 for both monkeys (1:200; Invitrogen). The slices were air dried overnight at room temperature, and then mounted with VECTASHIELD (Vector). The cell images were scanned using fluorescence microscope (Zeiss AXIO imager $\mathrm{M} 2)$. To test if the CDh-projecting neurons were dopaminergic, 
we examined co-localization of the TH signal and the remaining DY signal after the double labeling procedure.

\section{TOPOGRAPHICAL AND MORPHOLOGICAL ANALYSIS}

A major goal of this analysis was to create two kinds of 3D reconstruction: (1) the injection sites together with the boundary of $C D$, (2) the locations of labeled neurons together with the boundary of SN. For each reconstruction we first plotted these locations in each coronal slice using a microscope digitizer system that has an encoder attached to the microscope stage (AccuStage). We then aligned the coronal slice images to the corresponding $\mathrm{MR}$ images and created a stack of $2 \mathrm{D}$ coronal slices. For 3D reconstruction of injection sites, we used IMOD, a 3D rendering program (Boulder Laboratory, University of Colorado, Boulder, $\mathrm{CO}$, USA). For 3D reconstruction of labeled neurons in $\mathrm{SNc}$, we used a Matlab program (MathWorks). To quantitatively analyze the topographical segregation of $\mathrm{CDh}$-projecting neurons and CDt-projecting neurons, we used a discriminant analysis (Matlab, statistics toolbox).

A second goal was to analyze the morphology of CDhprojecting and CDt-projecting DA neurons. We plotted the cell soma of each CDh-projecting or CDt-projecting neuron based on TH immunoreactivity, and calculated its area and circularity using Image $(\mathrm{NIH})$, image processing and analysis program. We randomly sampled five projection neurons from each slice (500 $\mu \mathrm{m}$ interval) throughout SN of monkey ZO. The circularity was defined as follows: $4 \pi \times$ (cell area/perimeter 2$)$. A circularity value of 1.0 indicates a perfect circle, whereas a smaller value indicates a more elongated shape.

\section{RESULTS}

\section{DIFFERENCE IN OBJECT-VALUE LEARNING BETWEEN CDh AND CDt}

Our main question was whether DA neurons contribute differentially to the processing of flexible and stable values of visual objects in $\mathrm{CDh}$ and CDt. As a first step to answer this question, we studied the origins of DA inputs by injecting retrograde tracers in the flexible value-coding and stable value-coding regions. To determine the injection sites, we examined (1) where visual object-responsive neurons were located in $\mathrm{CD}$, and (2) whether these neurons encoded the values of the objects. In the first step, we confirmed our previous data: many neurons in $\mathrm{CDh}$ and $\mathrm{CDt}$ responded to visual stimuli (Hikosaka et al., 1989a; Yamamoto et al., 2012; Kim and Hikosaka, 2013). In CDt a majority of neurons were visually responsive; in $\mathrm{CDh}$ visually responsive neurons were often intermingled with non-visual neurons. Most CDt neurons responded to fractal objects differentially (i.e., object-selective); $\mathrm{CDh}$ neurons were less object-selective. In the second step, we examined whether these visually responsive neurons encoded flexible or stable values (Figure 1). The object values were created in two different ways (Kim and Hikosaka, 2013): (1) flexible values based on short-term object-reward association (Figure 1A), and (2) stable values based on long-term object-reward association (Figure 1C). Figures 1B,D show the responses of two example neurons, one in $\mathrm{CDh}$ and the other in $\mathrm{CDt}$, to fractal objects in the flexible value procedure (Figure 1B) and the stable value procedure (Figure 1D). They responded to the objects differentially with respect to their flexible or stable values.
In the short-term flexible value procedure (Figure 1A), two objects changed their values (i.e., associated with reward or no reward) across blocks of trials. The $\mathrm{CDh}$ neuron responded more strongly to the object that had recently been associated with reward (Figure 1B-left). In other words, the $\mathrm{CDh}$ neuron's response changed flexibly as an object changed its value. When two objects were presented simultaneously, the monkey made a saccade to the high-valued object in most trials to obtain the reward (Kim and Hikosaka, 2013). Thus, the CDh neuron's response was correlated with the monkey's choice preference among flexibly valued objects. In contrast, the CDt neuron responded to the two objects differently, but its responses were not affected by the flexible values of the objects (Figure 1B-right).

In the long-term stable value procedure, many objects retained their values stably during repeated learning across days (Figure 1C-top). To test the neuronal response, we presented the objects without reward feedback (Figure 1C-bottom). Thus, the values of these objects were solely based on long-term objectvalue learning. The $\mathrm{CDt}$ neuron overall responded more strongly to the high-valued objects than low-valued objects, although its response varied across objects (Figure 1D-right). When these objects were presented simultaneously, the monkey made saccades to high-valued objects even when no reward was given (Kim and Hikosaka, 2013). Thus, the CDt neuron's response was correlated with the monkey's choice preference among stably valued objects. In contrast, the $\mathrm{CDh}$ neuron showed no response to these objects, regardless of their stable values (Figure 1Dleft).

\section{RETROGRADE TRACER INJECTIONS IN CDh-FLEXIBLE AND CDt-STABLE VALUE LEARNING SITES}

The difference in object value-coding shown in Figures 1B,D was common among visually responsive neurons in $\mathrm{CDh}$ and $\mathrm{CDt}$ (Figures 2A,B), confirming our previous results (Kim and Hikosaka, 2013). However, CD contains many neurons that do not respond to visual stimuli but are sensitive to goal-directed behavior, especially in CDh (Hikosaka et al., 1989b). Therefore, to examine the role of DA neuron in object value learning, it was critical to determine the locations in each of $\mathrm{CDh}$ and $\mathrm{CDt}$ where the object value-coding neurons are most abundant. To answer this question, we explored the entire $\mathrm{CD}$ and identified neurons showing a statistically significant response bias with respect to the flexible or stable values. The distribution and density of such object value-coding neurons are shown in Figure 2C (flexible values) and Figure 2D (stable values).

The results indicate that the flexible value-coding neurons were relatively localized in the central region of $\mathrm{CDh}$ (Figure 2C). The stable value-coding neurons were commonly found in $\mathrm{CDt}$, except for the most caudal portion (Figure 2D). These CDh and CDt sites were critical for gaze orienting behavior based on flexible and stable value learning: using one of the monkeys (monkey ZO), we previously showed that inactivation of $\mathrm{CDh}$ (red "X" mark in Figure 2C) induced a selective impairment in the flexible valueguided behavior (controlled behavior), whereas inactivation of CDt (red "X" mark in Figure 2D) induced a selective impairment in the stable value-guided behavior (controlled behavior; Kim and Hikosaka, 2013). 


\section{A Short-term flexible value procedure}

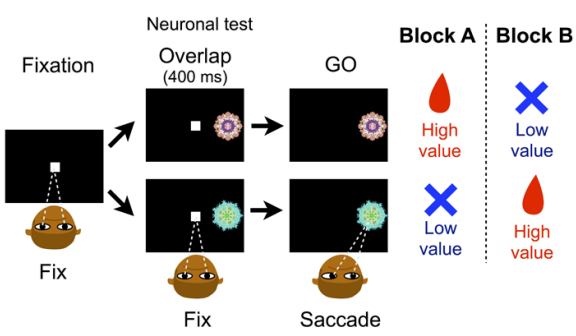

C

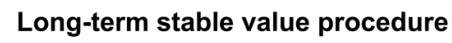

Learning long-term stable object values

High valued objects

Low valued objects

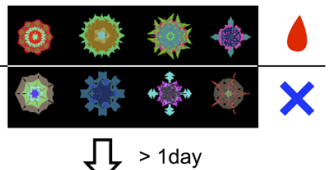
\> > day

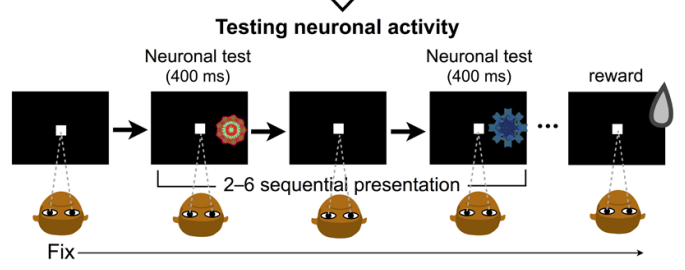

FIGURE 1 | Responses of CDh and CDt neurons to visual objects with flexible and stable values. (A) In the short-term flexible value procedure, each of two fractal objects changed its value frequently across blocks of trials. On each trial one of two objects was presented, to which monkeys made a saccade, which was followed by a liquid reward or no reward. Neuronal recording was done simultaneously. (B) Reponses of one CDh neuron (left) and one CDt neuron (right) in the flexible value procedure. The CDh neuron responded each object more strongly when the object was flexibly high-valued (red) than low-valued (blue; $p<0.05$, ranksum test). The CDt neuron responded to the two objects differently, but its response to each object was not affected by the flexible value changes. (C) In the long-term

D
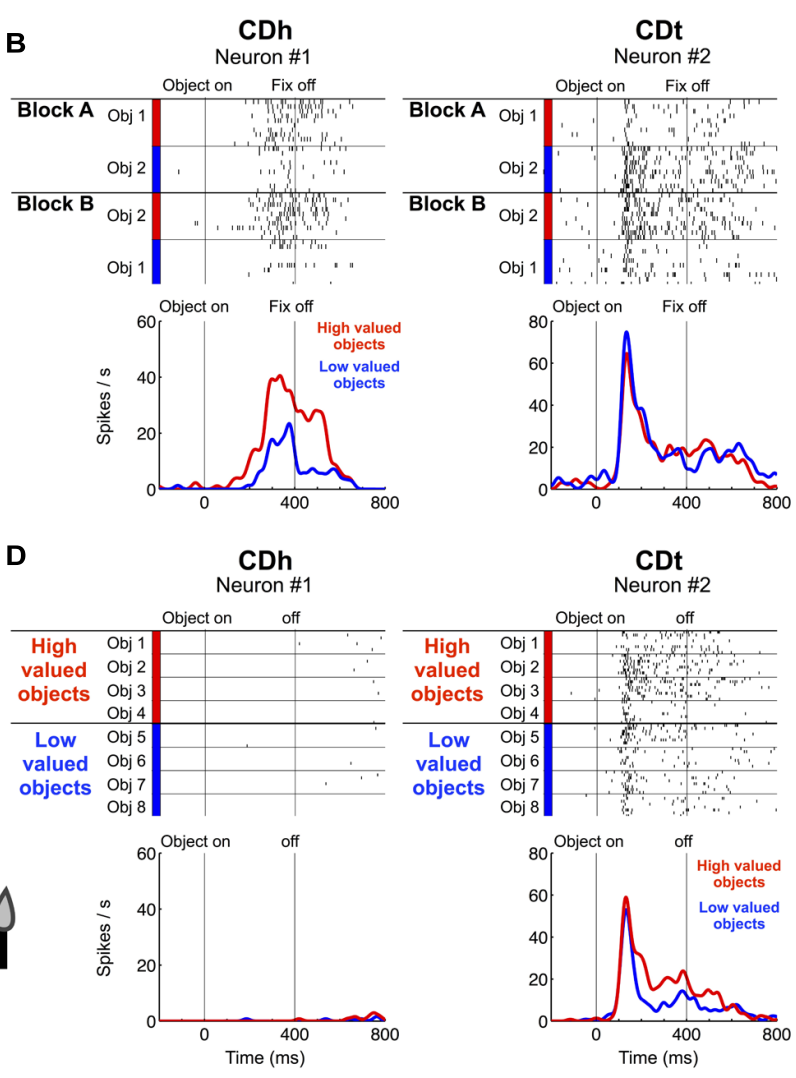

stable value procedure, each fractal object was associated with a high value (reward) or a low value (no reward) consistently during learning across days. The monkey experienced many sets of objects (eight fractals each), half high-valued and the other half low-valued (top). After the learning procedure, neuronal activity was tested on separate days using a fixation task in which the objects were presented one at a time with no contingent reward feedback (bottom). (D) Responses of the same CDh and CDt neurons as in (B). The CDt neuron (right) responded more strongly to the stably high-valued objects (red) than stably low-valued objects (blue), although it showed some object selectivity ( $p<0.05$, ranksum test). The CDh neuron (left) showed little response to the stably valued objects.
Based on the functional mapping, we injected retrograde tracers into the object value-coding sites in CDh and CDt (arrowheads in Figures 2C,D). Since the CDt site extends rostrocaudally, we injected tracers at two sites: rostral site $[\mathrm{CDt}(\mathrm{r})]$ and intermediate site $[\mathrm{CDt}(\mathrm{i})]$. To examine the specific projections of DA neurons into $\mathrm{CDh}$ and $\mathrm{CDt}$, different retrograde tracers were injected into the CDh and CDt (Table 1). Figure 3 shows the injection sites and labeling patterns in monkey SM. Diamidino Yellow (DY) was injected into the flexible value-coding domain in the central region of CDh (Figures 3A,B-left). Cholera toxin B subunit conjugated with Alexa-555 dye (CTB555) and FB were injected into the $\mathrm{CDt}(\mathrm{i})$ and $\mathrm{CDt}(\mathrm{r})$ respectively (Figures 3A,B-middle and right).

\section{DISTRIBUTION OF CDh- AND CDt-PROJECTING NEURONS IN SNC}

In the midbrain, retrogradely labeled neurons were found mainly in the ipsilateral substantia nigra pars compacta (SNc), but few in the ventral tegmental area (VTA) and contralateral SNc. Notably, neurons projecting to $\mathrm{CDh}$ and $\mathrm{CDt}$ were clustered in distinct regions of SNc. An example is shown in Figure 3C. CDhprojecting neurons were localized mainly in the ventral-medial region of SNc (yellow dots in Figure 3C), whereas CDt-projecting neurons were localized mainly in the dorsal-lateral region of SNc (cyan and red dots in Figure 3C). The CDh- and CDt-projecting areas (white boxes in Figure 3C) are enlarged in Figures 3D,E. The CDh-projecting area (ventral-medial SNc) contained DYlabeled cell nuclei (Figure 3D-left), but not CTB555-labled cell bodies (Figure 3D-right). The CDt-projecting area (dorsal-lateral SNc) contained CTB555-labled cell bodies (Figure 3E-right), but not DY-labeled cell nuclei (Figure 3E-left). These data suggested that separate groups of SNc neurons project to $\mathrm{CDh}$ and CDt.

Adjacent to the CDt-projecting area of SNc, we found a CTB55labeled plexus which presumably represents anterogradely labeled axonal terminals of CDt neurons (Figure 3E-right). The plexus was located in the dorsal-lateral SNr. The result suggests that 
A

\section{Short-term \\ flexible value coding}

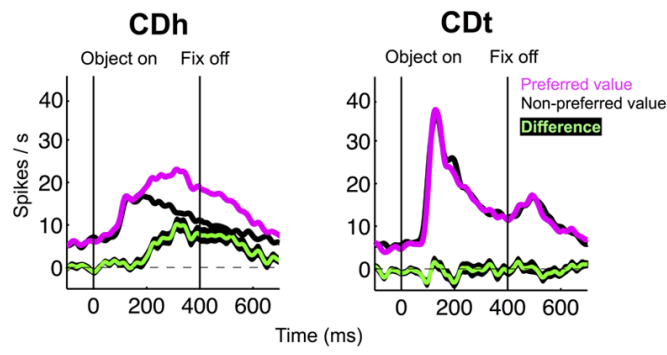

C

Distribution of flexible value-coding neurons

Sagittal section of caudate nucleus

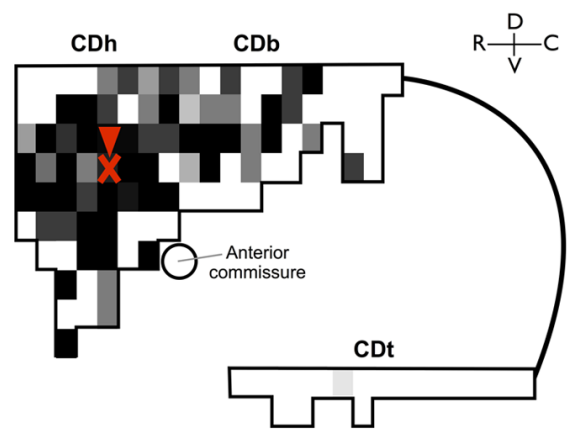

FIGURE 2 |Value-coding map of CD. (A) Average responses to the flexibly valued objects of neurons in CDh $(n=71)$ and CDt $(n=54)$. Neuronal responses were averaged for the neurons' preferred values (magenta) and non-preferred values (black) using a cross-validation method. The green line indicates the mean of the differences between the preferred and non-preferred responses, and the black line behind the green line indicates the standard error of the differences (mean $\pm S E$ ). (B) Average responses to the stably valued objects of neurons in CDh $(n=32)$ and CDt $(n=59)$. Same format as in $(\mathbf{A})$. Data in $(\mathbf{A}, \mathbf{B})$ were
B

\section{Long-term stable value coding}

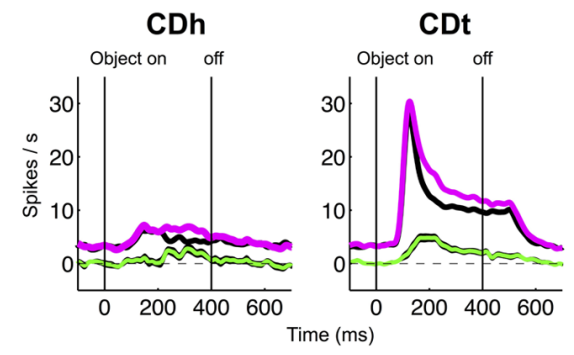

D

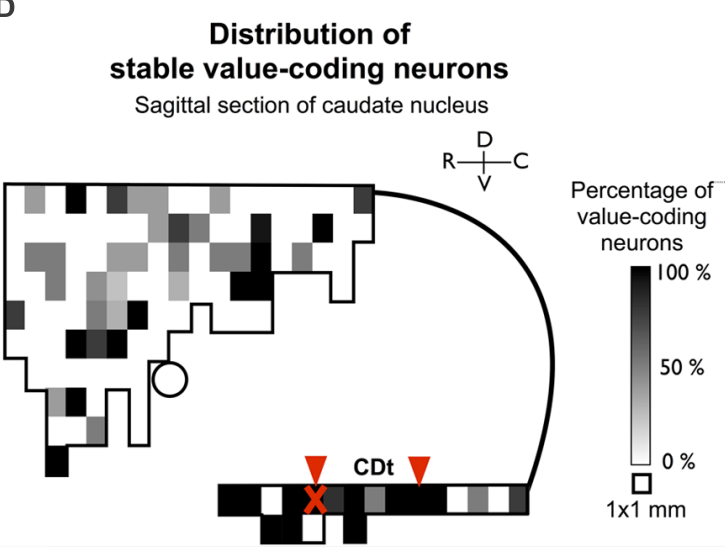

Table 1 | Retrograde tracers injected into CDh or CDt.

\begin{tabular}{llll}
\hline Monkey & $\begin{array}{l}\text { CDh injection } \\
\text { (Short-term flexible value-coding) }\end{array}$ & $\begin{array}{l}\text { CDt(r) injection } \\
\text { (Long-term stable value-coding) }\end{array}$ \\
\hline SM & Diamidino Yellow (DY) & Fast Blue (FB) \\
ZO & Diamidino Yellow (DY) & Cholera Toxin-Alexa488 (CTB488)
\end{tabular}

Different kinds of retrograde tracers were injected into CDh, CDt(r) and CDt(i).

CDt neurons have direct output connections to dorsal-lateral $\mathrm{SNr}$ neurons and receive direct inputs from dorsal-lateral SNc neurons.

The dissociation of CDh- and CDt-projecting neurons was found in the entire SNc of monkey SM (Figure 4A). They were largely segregated in all of the three dimensional axes (Figure 4Bleft): rostral-ventral-medial ( $\mathrm{r}-\mathrm{v}-\mathrm{m}) \mathrm{SNc}$ neurons projected to $\mathrm{CDh}$, whereas caudal-dorsal-lateral (c-d-l) SNc neurons projected to CDt. The segregation of CDh- and CDt-projecting neurons in each axis of $\mathrm{SN}$ was statistically significant $(p<0.001$, twotailed $t$-test; Figure 4B-right). Discriminant analysis showed that $\mathrm{CDh}$ - and CDt-projecting neurons were largely separated into two groups by a single quadratic plane (green discriminant plane in Figure 4C; Table 2).

We confirmed the same tendency in monkey ZO (Figure 5). DY was injected into the central part of CDh (yellow in Figures 5A,B). CTB555 and CTB488 (cholera toxin B subunit conjugated with Alexa-488 dye) were injected into $\mathrm{CDt}(\mathrm{i})$ and $\mathrm{CDt}(\mathrm{r})$ respectively (red and green in Figures 5A,B). CDh- and CDt-projecting neurons were separately localized in $\mathrm{r}-\mathrm{v}-\mathrm{m} \mathrm{SNc}$ and c-d-1 SNc, similarly to the distributions in monkey SM (Figures 5C-E). Notably, neurons projecting to both $\mathrm{CDh}$ and $\mathrm{CDt}$ were rarely found in monkey SM and ZO (Table 3 ). In addition, the $\operatorname{CDt}(\mathrm{r})-$ and 
A

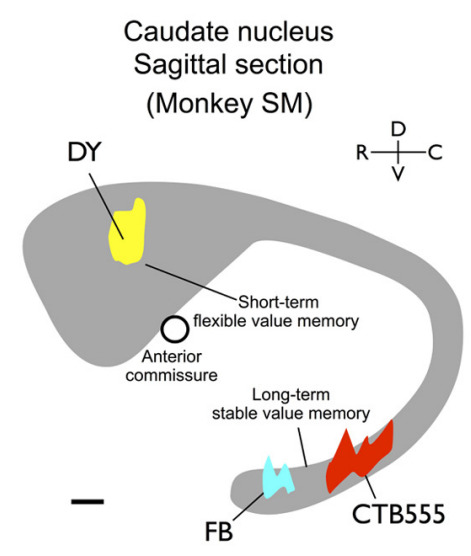

B

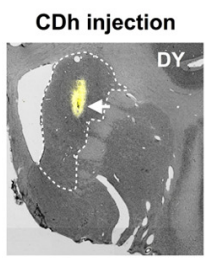

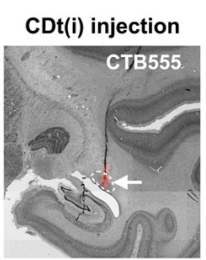

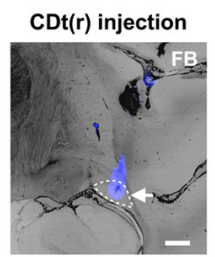

C

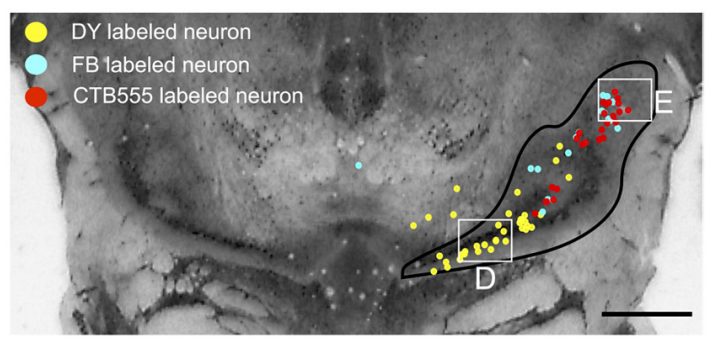

D
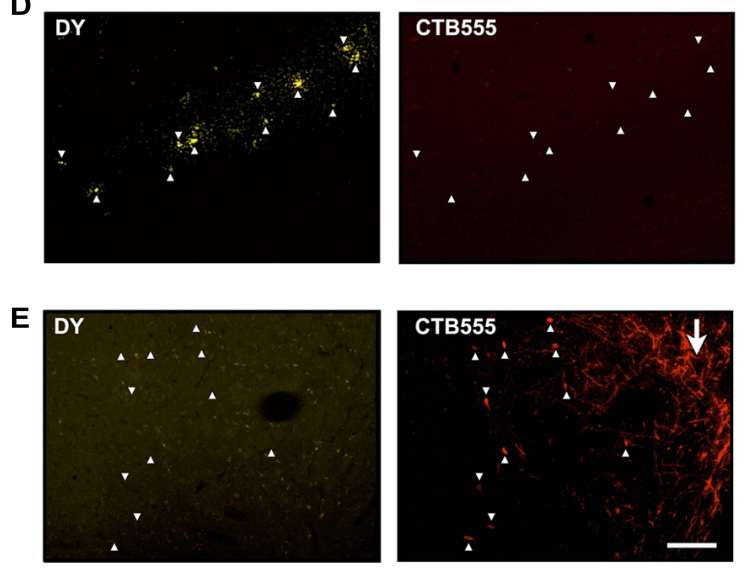

FIGURE 3 | Retrograde tracer injections in two functional domains of CD. (A) Side view of CD showing injection sites of DY (in CDh), CTB555 and FB (in CDt) in monkey SM. Circle: anterior commissure. Scale bar: $2 \mathrm{~mm}$. (B) Nissl-stained coronal sections showing the injection sites. White dotted lines indicate the borders of CDh and CDt. Scale bar: $2 \mathrm{~mm}$. (C) Retrogradely labeled neurons in and around SN (Black line).
Boxed regions are examined in (D,E). Scale bar: $5 \mathrm{~mm}$. (D) DY-labeled CDh-projecting neurons (left, indicated by white arrowheads) were not labeled with CTB555 (right). (E) CTB555-labeled CDt-projecting neurons (right) were not labeled with DY (left). White arrow indicates axon terminals of CDt neurons labeled with CTB555 anterogradely (right). Scale bar: $200 \mu \mathrm{m}$.
CDt(i)-projecting neurons were mostly separated, although they were similarly localized in c-d-1 SNc (Table 4; Figures 4B and 5D). These data indicated that separate groups of neurons in $\mathrm{r}-\mathrm{v}-\mathrm{m} \mathrm{SNc}$ and $\mathrm{c}-\mathrm{d}-\mathrm{l} \mathrm{SNc}$ innervated the flexible value-coding $\mathrm{CDh}$ and the stable value-coding CDt.

\section{MORPHOLOGICAL DIFFERENCE OF TH-POSITIVE DA NEURONS IN r-v-m AND c-d-I SNc}

To test if the retrogradely labeled neurons are dopaminergic, we double-labeled SN sections (500 $\mu \mathrm{m}$ interval) with antibodies against CTB and DA-specific marker protein, TH (Figure 6). Anti-CTB antibody labeled the CDt-projecting neurons in SNc (Figure 6A, top-right, red dots). Anti-TH antibody labeled the cell soma and dendrites/axons of DA neurons (Figure 6A, bottom-right, green signals). Importantly, all the CTB-positive neurons (Figure 6A, top-right) were TH-positive (Figure 6A, bottom-right); they were double labeled (Figure 6A, bottomleft, yellow dots). This was true throughout the sections of SN, indicating that the CDt-projecting neurons were dopaminergic. The proportions of CDh- and CDt-projecting neurons among TH-positive neurons were 2.16 and $0.40 \%$ in monkey SM and 0.75 and $0.33 \%$ in monkey ZO. The CTB-labeled axon terminals of CDt neurons (Figure 6A, top-left and top-right, red plexus) were located in $\mathrm{SNr}$ and did not overlap with CDtprojecting DA neurons. CDh-projecting neurons were also likely to be dopaminergic because DY-positive neurons were TH-positive (Figure 6B-left). Notably, these CDh- and CDt-projecting DA neurons were different in morphological features. We compared the size and shape of cell bodies between DY and TH double positive neurons (Figure 6B-left) and CTB555-TH double positive neurons (Figure 6B-right): CDt-projecting DA neurons were larger but less circular than $\mathrm{CDh}$-projecting DA neurons (Figure 6C).

\section{DISCUSSION}

\section{TOPOGRAPHICAL ORGANIZATION OF DOPAMINE INNERVATION IN THE} STRIATUM

Our retrograde tracer experiments suggested that largely separate groups of DA neurons in the monkey SNc (r-v-m SNc and c-d$1 \mathrm{SNc}$ ) innervate $\mathrm{CDh}$ and $\mathrm{CDt}$. It is possible that some of the retrograde labeling occurred via damaged axons en passage, especially because electrodes for neuronal recording were penetrated multiple times before the tracer injections. However, it is unlikely that such erroneous labeling heavily affected the results, because the retrograde labeling pattern inside SNc was similar between the two monkeys and the tracers were mainly placed inside CD.

Our result is consistent with the concept that DA innervation in the striatum is topographically organized: DA neurons in different regions of the SNc and VTA project to different regions of the striatum in monkeys and rodents (Haber 


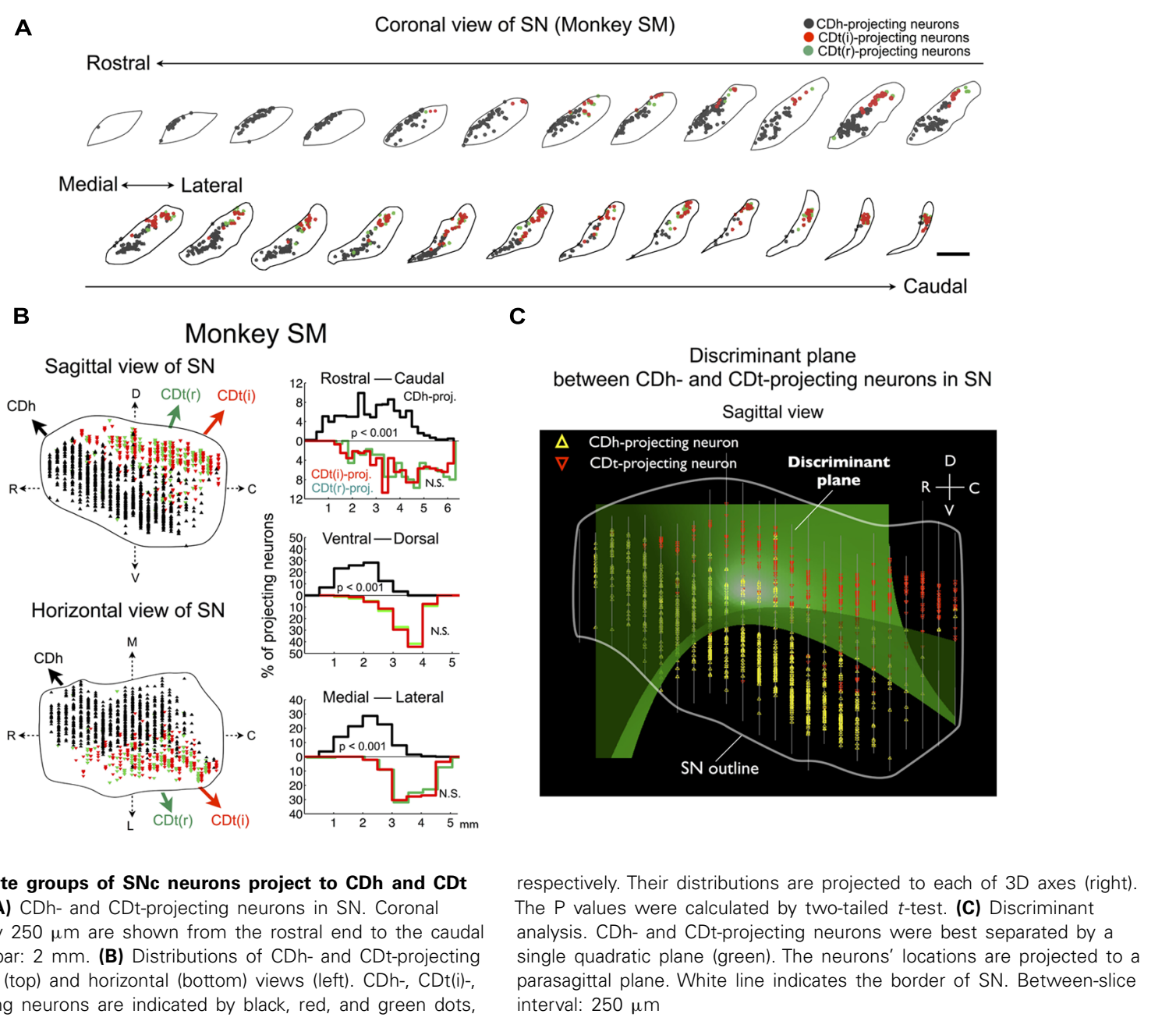

Table 2 | Proportions of CDh and CDt-projecting neurons in two regions of SNc separated by a discriminant plane.

\section{CDh-projecting (\%) CDt-projecting (\%)}

\begin{tabular}{lll}
\hline Monkey SM & & \\
Group 1(c-d-l SNc) & 8.4 & 88.9 \\
Group 2(r-v-m SNc) & 91.6 & 11.1 \\
Monkey ZO & & \\
Group 1(c-d-l SNc) & 10.2 & 83.0 \\
Group 2(r-v-m SNc) & 89.8 & 17.0 \\
\hline
\end{tabular}

Two groups of CDh- and CDt-projecting DA neurons are spatially separated by a discriminant analysis. Percentage indicates the proportion of CDh- and CDtprojecting neurons inside each discriminant plane (green plane in Figures $\mathbf{4 C}$ and $5 E)$.

et al., 2000; Joel and Weiner, 2000; Ikemoto, 2007). Notably, most anatomical studies have focused on the dorsal-ventral or medial-lateral difference in the striatum, and rarely examined the rostral-caudal difference. This is a missing link for DA functions, because, in monkeys and humans, the rostral-caudal regions of the striatum have been shown to participate in procedural learning at different stages (Hikosaka et al., 1999; Floyer-Lea and Matthews, 2004; Lehéricy et al., 2005; Tricomi et al., 2009; Seger etal., 2010) and action selection in different manners (Jankowski etal., 2009). In particular, the projections of DA neurons to subregions of CD, including CDt, have not been studied. Since CDt is prominent in primates (Hjornevik et al., 2007), a comparison of DA innervation between CDh and CDt may reveal a unique function of the primate DA system including humans.

Previous anatomical studies have shown that different groups of DA neurons innervate different regions of the striatum. However, it is unclear whether the differential DA innervation affects motor behavior differentially. Our results suggest that separate DA innervation controls a common motor output (i.e., gaze orienting) because $\mathrm{CDh}$ and $\mathrm{CDt}$ have downstream connections to SC through SNr (Hikosaka et al., 2000; Yamamoto et al., 2012; Yasuda et al., 2012). This suggests that a single motor action can be controlled differently by separate DA innervation. Indeed, our previous studies indicated that gaze orienting is influenced by the reward value of the target object in two different ways, which are processed in CDh and CDt separately (Kim and Hikosaka, 2013). Below, we will discuss this point further. 
Table 3 | Numbers of SNc neurons projecting to $\mathrm{CDh}, \mathrm{CDt}$, and both.

\begin{tabular}{llllll}
\hline Monkey & CDh-projecting(\# of cell) & CDt-projecting(\# of cell) & Both(\# of cell) & Both/CDh-projecting (\%) & Both/CDt-projecting (\%) \\
\hline SM & 1059 & 455 & 9 & 0.85 & 1.98 \\
ZO & 557 & 270 & 4 & 0.72 & 1.48 \\
\hline
\end{tabular}

Numbers indicate CDh-, CDt-, and both projecting neurons. Percentage indicates the proportion of both projecting neurons within CDh-projecting or CDt-projecting neurons.

Table 4 | Numbers of SNc neurons projecting to rostral CDt, intermediate CDt, and both.

\begin{tabular}{llllll}
\hline Monkey & $\mathbf{C D t}(\mathbf{r})$-projecting(\# of cell) & $\mathbf{C D t}(\mathbf{i})$-projecting(\# of cell) & Both(\# of cell) & Both/CDt(r)-projecting (\%) & Both/CDt(i)-projecting (\%) \\
\hline SM & 175 & 280 & 15 & 8.57 & 5.36 \\
ZO & 151 & 119 & 7 & 4.64 & 5.88
\end{tabular}

Numbers indicate CDt(r)-, CDt(i)-, and both projecting neurons. Percentage indicates the proportion of both projecting neurons within CDt(r)-projecting or CDt(i)projecting neurons.

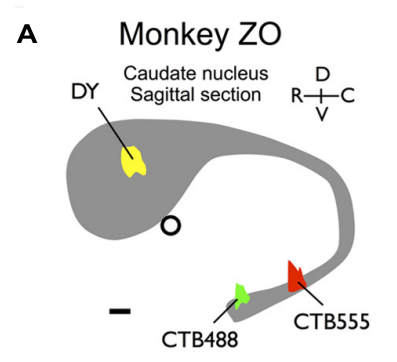

B

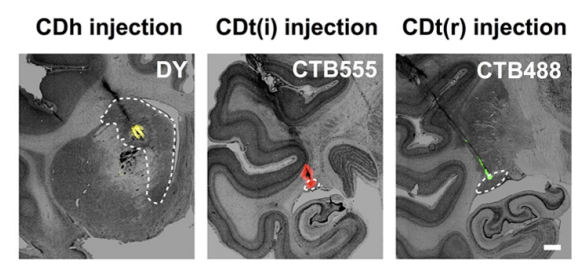

C

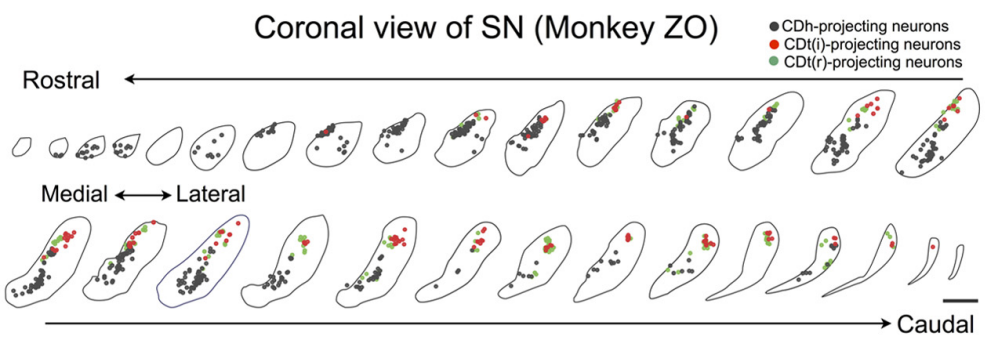

D

Monkey ZO

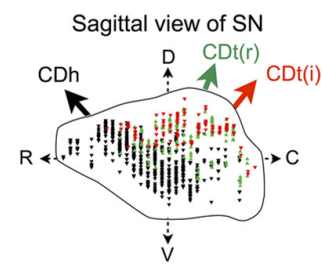

Horizontal view of SN

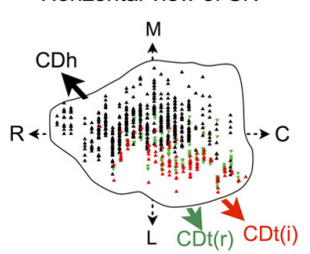

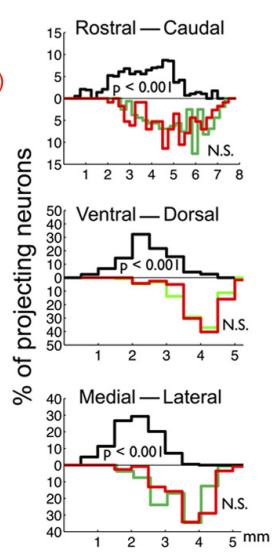

E between CDh- and CDt-projecting neurons in SN

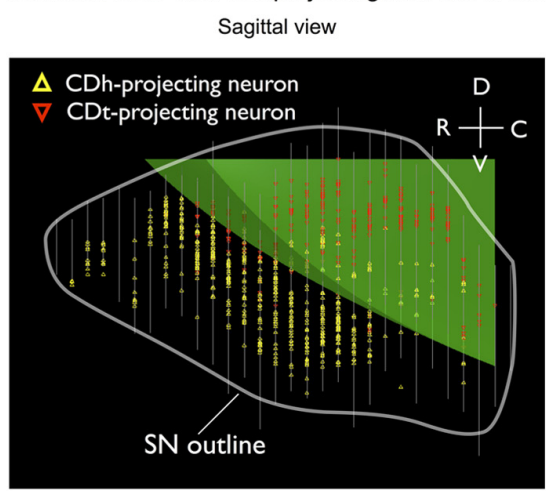

FIGURE 5 | Projections of SNc neurons to CDh and CDt in monkey ZO. Same format as in Figures $\mathbf{3}$ and $\mathbf{4}$. 

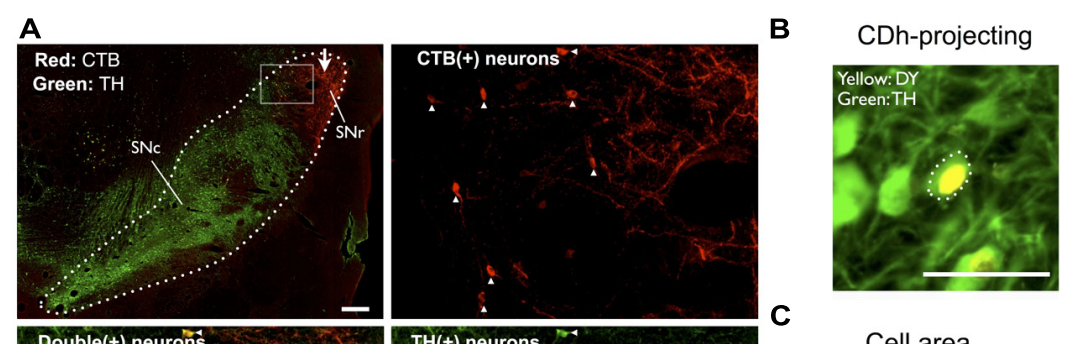

C
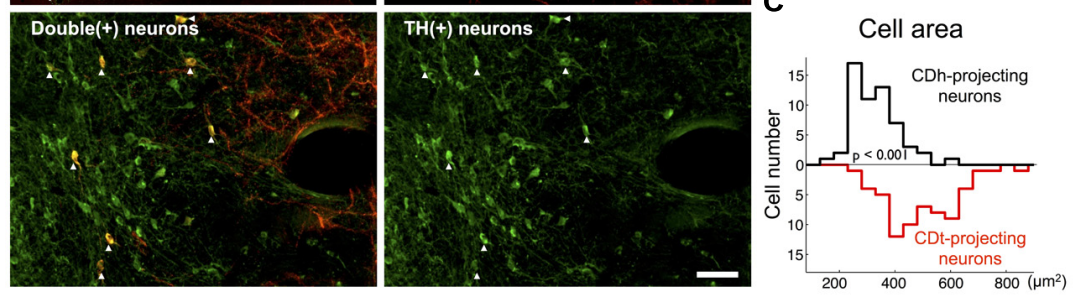

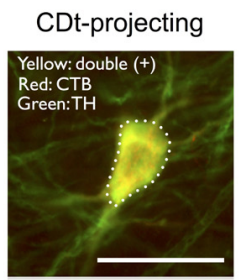

Circularity

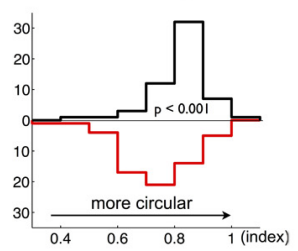

FIGURE 6 | CDt- and CDh-projecting neurons in SNc are dopaminergic. (A) Distributions of CDt-projecting neurons (CTB-positive, red) and DA neurons (TH-positive, green) in monkey SM. The CDt-projecting neurons were clustered in the dorsolateral part of $\mathrm{SN}$ (dotted line in top-left section; scale bar: $500 \mu \mathrm{m}$ ), and this area (box in top-left) is enlarged in the other sections: CTB-positive (top-right), TH-positive (bottom-right), and merged (bottom-left; scale bar: $100 \mu \mathrm{m}$ ). The merged image (bottom-left) shows double-labeled neurons (yellow signal). White arrow indicates CTB-labeled axon terminals of CDt neurons (top-left). (B) Difference in cell area and circularity of CDh- and CDt-projecting DA neurons. Sample CDh- and CDt-projecting DA neurons (top) in monkey ZO. DY signal was localized in the nucleus (top-left), while CTB and TH signals were distributed in the whole cell (top-right). Dotted line indicates cell soma. Scale bar: $50 \mu \mathrm{m}$. (C) Left: CDt-projecting DA neurons had larger cell areas than CDh-projecting neurons (mean value: 467.6 vs. $304.2 \mu \mathrm{m}^{2} ; p<0.001$, ranksum test; bottom-left). Right: CDh-projecting DA neurons were more circular than CDt-projecting neurons (mean value: 0.69 vs. $0.77 ; p<0.001$, ranksum test; bottom-right).

\section{DOPAMINE MECHANISMS UNDERLYING FAST AND SLOW-LEARNING}

Our data showed fast-learning and forgetting of object values in $\mathrm{CDh}$ neurons (flexible value-coding) and slow-learning and forgetting in CDt neurons (stable value-coding; Figures $\mathbf{1}$ and 2; also see Kim and Hikosaka, 2013). Furthermore, the different time courses of learning in $\mathrm{CDh}$ and CDt might be caused by their separate inputs from the two groups of DA neurons in r-v-m SNc and c-d-1 SNc. Below, we consider two hypothetical mechanisms.

A common concept of the DA mechanism is that the glutamatergic cortico-striatal synaptic transmission is enhanced or depressed by the repeated association or disassociation with DA inputs to the striatal neurons (Reynolds and Wickens, 2002; Surmeier et al., 2007). However, it is unclear how long the synaptic plasticity remains, especially during natural behavior. Our data, following the above concept, suggest that r-v-m SNc-DA neurons cause strong but short-lasting synaptic plasticity in the corticoCDh transmission, whereas c-d-l SNc-DA neurons cause weak but long-lasting synaptic plasticity in the cortico-CDh transmission (mechanism \#1). A critical question, then, would be: what determines the distinct time courses of the synaptic plasticity in CDh vs. CDt neurons. This remains to be addressed in future studies.

A simple assumption behind the above hypothesis is that both of the two groups of DA neurons send a common value signal, yet their outcomes are distinct (i.e., flexible vs. stable valuecoding) due to the different time courses of synaptic plasticity. Alternatively, the two groups of DA neurons might send different kinds of signal to $\mathrm{CDh}$ and $\mathrm{CDt}$, thereby causing the distinct value-codings (mechanism \#2). A well characterized signal conveyed by DA neurons is reward prediction error (RPE) (Schultz,
1998), which represents an instantaneous change of reward values (i.e., actual reward value - expected reward value) and therefore is suited to generate flexible value-coding. Then, mechanism \#2 predicts that $\mathrm{r}-\mathrm{v}-\mathrm{m}$ SNc-DA neurons encode RPE, but c-d-l SNcDA neurons do not. The hypothetical difference in value-coding might be related to the morphological differences in cell soma (Figures 6B,C): CDt-projecting DA neurons in c-d-1 SNc tended to be larger and less circular than CDh-projecting DA neurons in $\mathrm{r}-\mathrm{v}-\mathrm{m}$ SNc. Testing mechanism \#2 requires further experiments, particularly recording activity of CDt-projecting DA neurons in c-d-l SNc.

\section{CIRCUIT MECHANISMS}

The above hypothesis (mechanism \#2) attempts to explain the difference in learning and memory between CDh and CDt by DA inputs, which basically reassigns the question to DA neurons. The real solution could instead be found in neuronal circuits. Our data showed that CDt neurons project their axons selectively to the caudal-dorsal-lateral portion of the $\mathrm{SNr}$ (c-d-l SNr) which was adjacent to the CDt-projecting DA neurons in c-d-l SNc (Figure 6A). It has been shown that GABAergic SNr neurons have axon collaterals to adjacent GABAergic $\mathrm{SNr}$ neurons as well as DAergic SNc neurons (Deniau et al., 1982; Tepper et al., 1995). These findings together suggest a loop circuit [CDt(GABA) $\rightarrow$ $\mathrm{c}-\mathrm{d}-\mathrm{S} \mathrm{SNr}(\mathrm{GABA}) \rightarrow \mathrm{c}-\mathrm{d}-\mathrm{l} \mathrm{SNc}(\mathrm{DA}) \rightarrow \mathrm{CDt}]$. If this works as a positive loop, reward value signals might be retained through the loop circuit, providing a basis of long-term object-value memories.

A similar loop circuit may be formed for CDh-projecting DA neurons [i.e., $\mathrm{CDh}(\mathrm{GABA}) \rightarrow \mathrm{r}-\mathrm{v}-\mathrm{m} \mathrm{SNr}(\mathrm{GABA}) \rightarrow \mathrm{r}-\mathrm{v}-\mathrm{m}$ $\mathrm{SNc}(\mathrm{DA}) \rightarrow \mathrm{CDh}]$, because the distribution of CDh-projecting 
neurons (Figures 4 and 5) roughly matches the portion of $\mathrm{SNr}$ that receive inputs from $\mathrm{CDh}$ reported previously (Smith and Parent, 1986; Hikosaka et al., 1993). If this CDh loop circuit works similarly to CDt loop circuit (suggested above), it would also promote long-term object-value memories, but apparently it is not the case. This question remains an interesting issue for future research.

\section{IMPLICATIONS FOR DOPAMINE DISORDERS}

Consistent with anatomical data described above, CDh-derived flexible value signals and CDt-derived stable value signals are mediated by different regions in $\mathrm{SNr}$ (c-d-l SNr and r-v-m SNr, respectively), both of which project to SC (Yasuda and Hikosaka, 2013). Experimental inactivation of the parallel circuits disrupted value-based gaze orienting, but in different manners (Kim and Hikosaka, 2013): CDh inactivation disrupted the preferred gaze orienting to objects that were immediately followed by a large reward (i.e., controlled orienting), whereas CDt inactivation disrupted the preferred gaze orienting to objects that had previously been associated with a large reward repeatedly but not currently (i.e., automatic orienting). This suggests the dichotomy of DA function: CDh-projecting DA neurons guide controlled behavior, whereas CDt-projecting DA neurons guide automatic behavior.

This suggestion provides an important clue to understanding DA dysfunctions. Patients with Parkinson's disease may be impaired in cognitive and/or automatic behaviors (Brown and Marsden, 1990; Redgrave et al., 2010). Our study raises the possibility that impairments in cognitive behaviors may be caused by the degeneration of CDh-projecting DA neurons in r-v-m SNc, whereas impairments in automatic behaviors may be caused the degeneration of CDt-projecting DA neurons in c-d-1 SNc.

In Parkinson's disease, the degeneration of DA neurons tends to occur first in the lateral part of SNc (Rinne et al., 1989; Fearnley and Lees, 1991). We showed that larger and less circular DA neurons were localized in this lateral SNc (Figure 6B), suggesting that this type of DA neurons might be more vulnerable to cell death in Parkinson's disease. Our data may provide an opportunity for differential diagnosis and treatments of Parkinson's disease and other DA disorders.

\section{AUTHOR CONTRIBUTIONS}

All authors, Hyoung F. Kim, Ali Ghazizadeh, and Okihide Hikosaka meet the four criteria for authorship.

\section{ACKNOWLEDGMENTS}

We express special thanks to K. S. Saleem and M. K. Smith for technical assistance and suggestions. We also thank M. Yasuda, I. Monosov, and E. Bromberg-Martin for discussions and D. Parker, I. Bunea, G.Tansey, A.M. Nichols, T. W. Ruffner, J. W. McClurkin, and A. V. Hays for technical assistance. This research was supported by the Intramural Research Program at the National Institutes of Health - National Eye Institute.

\section{REFERENCES}

Alexander, G. E., DeLong, M. R., and Strick, P. L. (1986). Parallel organization of functionally segregated circuits linking basal ganglia and cortex. Annu. Rev. Neurosci. 9, 357-381. doi: 10.1146/annurev.ne.09.030186.002041
Brown, R. G., and Marsden, C. D. (1990). Cognitive function in Parkinson's disease: from description to theory. Trends Neurosci. 13, 21-29. doi: 10.1016/0166-2236(90)90058-I

Deniau, J. M., Kitai, S. T., Donoghue, J. P., and Grofova, I. (1982). Neuronal interactions in the substantia nigra pars reticulata through axon collaterals of the projection neurons. An electrophysiological and morphological study. Exp. Brain Res. 47, 105-113. doi: 10.1007/BF00235891

Fearnley, J. M., and Lees, A. J. (1991). Ageing and Parkinson'S disease: substantia nigra regional selectivity. Brain 114, 2283-2301. doi: 10.1093/brain/114. 5.2283

Floyer-Lea, A., and Matthews, P. M. (2004). Changing brain networks for visuomotor control with increased movement automaticity. J. Neurophysiol. 92, 2405-2412. doi: 10.1152/jn.01092.2003

Frank, M. J., Seeberger, L. C., and O'reilly, R. C. (2004). By carrot or by stick: cognitive reinforcement learning in parkinsonism. Science 306, 1940-1943. doi: 10.1126/science.1102941

Haber, S. N., Fudge, J. L., and McFarland, N. R. (2000). Striatonigrostriatal pathways in primates form an ascending spiral from the shell to the dorsolateral striatum. J. Neurosci. 20, 2369-2382.

Hikosaka, O., Kim, H. F., Yasuda, M., and Yamamoto, S. (2014). Basal ganglia circuits for reward value - guided behavior. Annu. Rev. Neurosci. 37, 289-306. doi: 10.1146/annurev-neuro-071013-013924

Hikosaka, O., Nakahara, H., Rand, M. K., Sakai, K., Lu, X., Nakamura, K., et al. (1999). Parallel neural networks for learning sequential procedures. Trends Neurosci. 22, 464-471. doi: 10.1016/S0166-2236(99)01439-3

Hikosaka, O., Sakamoto, M., and Miyashita, N. (1993). Effects of caudate nucleus stimulation on substantia nigra cell activity in monkey. Exp. Brain Res. 95, 457472. doi: 10.1007/BF00227139

Hikosaka, O., Sakamoto, M., and Usui, S. (1989a). Functional properties of monkey caudate neurons. II. Visual and auditory responses. J. Neurophysiol. 61, 799-813.

Hikosaka, O., Sakamoto, M., and Usui, S. (1989b). Functional properties of monkey caudate neurons. III. Activities related to expectation of target and reward. J. Neurophysiol. 61, 814-832.

Hikosaka, O., Takikawa, Y., and Kawagoe, R. (2000). Role of the basal ganglia in the control of purposive saccadic eye movements. Physiol. Rev. 80, 953-978.

Hjornevik, T., Leergaard, T. B., Darine, D., Moldestad, O., Dale, A. M., Willoch, F., et al. (2007). Three-dimensional atlas system for mouse and rat brain imaging data. Front. Neuroinform. 1:4. doi: 10.3389/neuro.11.004.2007

Ikemoto, S. (2007). Dopamine reward circuitry: two projection systems from the ventral midbrain to the nucleus accumbens-olfactory tubercle complex. Brain Res. Rev. 56, 27-78. doi: 10.1016/j.brainresrev.2007.05.004

Jankowski, J., Scheef, L., Hüppe, C., and Boecker, H. (2009). Distinct striatal regions for planning and executing novel and automated movement sequences. Neuroimage 44, 1369-1379. doi: 10.1016/j.neuroimage.2008.10.059

Joel, D., and Weiner, I. (2000). The connections of the dopaminergic system with the striatum in rats and primates: an analysis with respect to the functional and compartmental organization of the striatum. Neuroscience 96, 451-474. doi: 10.1016/S0306-4522(99)00575-8

Kato, M., Miyashita, N., Hikosaka, O., Matsumura, M., Usui, S., and Kori, A. (1995). Eye movements in monkeys with local dopamine depletion in the caudate nucleus. I. Deficits in spontaneous saccades. J. Neurosci. 15(1 Pt 2), 912-927.

Kim, H. F., and Hikosaka, O. (2013). Distinct basal ganglia circuits controlling behaviors guided by flexible and stable values. Neuron 79, 1001-1010. doi: 10.1016/j.neuron.2013.06.044

Kravitz, A. V., Freeze, B. S., Parker, P. R. L., Kay, K., Thwin, M. T., Deisseroth, K., etal. (2010). Regulation of parkinsonian motor behaviours by optogenetic control of basal ganglia circuitry. Nature 466, 622-626. doi: 10.1038/ nature09159

Lehéricy, S., Benali, H., Van de Moortele, P.-F., Pélégrini-Issac, M., Waechter, T., Ugurbil, K., et al. (2005). Distinct basal ganglia territories are engaged in early and advanced motor sequence learning. Proc. Natl. Acad. Sci. U.S.A. 102, 1256612571. doi: 10.1073/pnas.0502762102

McHaffie, J. G., Stanford, T. R., Stein, B. E., Coizet, V., and Redgrave, P. (2005). Subcortical loops through the basal ganglia. Trends Neurosci. 28, 401-407. doi: 10.1016/j.tins.2005.06.006

Mink, J. W. (1996). The basal ganglia: focused selection and inhibition of competing motor programs. Prog. Neurobiol. 50, 381-425. doi: 10.1016/S03010082(96)00042-1 
Miyachi, S., Hikosaka, O., and Lu, X. (2002). Differential activation of monkey striatal neurons in the early and late stages of procedural learning. Exp. Brain Res. 146, 122-126. doi: 10.1007/s00221-002-1213-7

Miyachi, S., Hikosaka, O., Miyashita, K., Kárádi, Z., and Rand, M. K. (1997). Differential roles of monkey striatum in learning of sequential hand movement Exp. Brain Res. 115, 1-5.

Parent, A., and Hazrati, L. N. (1995). Functional anatomy of the basal ganglia. II. The place of subthalamic nucleus and external pallidum in basal ganglia circuitry. Brain Res. Brain Res. Rev. 20, 128-154.

Redgrave, P., Rodriguez, M., Smith, Y., Rodriguez-Oroz, M. C., Lehericy, S., Bergman, H., et al. (2010). Goal-directed and habitual control in the basal ganglia: implications for Parkinson's disease. Nat. Rev. Neurosci. 11, 760-772. doi: $10.1038 / \mathrm{nrn} 2915$

Reynolds, J. N., and Wickens, J. R. (2002). Dopamine-dependent plasticity of corticostriatal synapses. Neural Netw. 15, 507-521. doi: 10.1016/S0893 6080(02)00045-X

Richfield, E. K., Young, A. B., and Penney, J. B. (1987). Comparative distribution of dopamine D-1 and D-2 receptors in the basal ganglia of turtles, pigeons, rats, cats, and monkeys. J. Comp. Neurol. 262, 446-463. doi: 10.1002/cne.902620308

Rinne, J. O., Rummukainen, J., Paljärvi, L., and Rinne, U. K. (1989). Dementia in Parkinson's disease is related to neuronal loss in the medial substantia nigra. Ann. Neurol. 26, 47-50. doi: 10.1002/ana.410260107

Saint-Cyr, J. A., Ungerleider, L. G., and Desimone, R. (1990). Organization of visual cortical inputs to the striatum and subsequent outputs to the pallido-nigral complex in the monkey. J. Comp. Neurol. 298, 129-156. doi 10.1002/cne.902980202

Schultz, W. (1998). Predictive reward signal of dopamine neurons. J. Neurophysiol. $80,1-27$.

Seger, C. A., Peterson, E. J., Cincotta, C. M., Lopez-Paniagua, D., and Anderson, C. W. (2010). Dissociating the contributions of independent corticostriatal systems to visual categorization learning through the use of reinforcement learning modeling and Granger causality modeling. Neuroimage 50, 644-656. doi: 10.1016/j.neuroimage.2009.11.083

Selemon, L. D., and Goldman-Rakic, P. S. (1985). Longitudinal topography and interdigitation of corticostriatal projections in the rhesus monkey. J. Neurosci. 5 , 776-794.

Smith, Y., and Parent, A. (1986). Differential connections of caudate nucleus and putamen in the squirrel monkey (Saimiri sciureus). Neuroscience 18, 347-371. doi: 10.1016/0306-4522(86)90159-4

Surmeier, D. J., Ding, J., Day, M., Wang, Z., and Shen, W. (2007). D1 and D2 dopamine-receptor modulation of striatal glutamatergic signaling in striatal medium spiny neurons. Trends Neurosci. 30, 228-235. doi: 10.1016/j.tins.2007.03.008

Tepper, J. M., Martin, L. P., and Anderson, D. R. (1995). GABA receptor-mediated inhibition of rat substantia nigra dopaminergic neurons by pars reticulata projection neurons. J. Neurosci. 15, 3092-3103.

Tricomi, E., Balleine, B. W., and O'Doherty, J. P. (2009). A specific role for posterior dorsolateral striatum in human habit learning. Eur. J. Neurosci. 29, 2225-2232. doi: 10.1111/j.1460-9568.2009.06796.x

Yamamoto, S., Kim, H. F., and Hikosaka, O. (2013). Reward value-contingent changes of visual responses in the primate caudate tail associated with a visuomotor skill. J. Neurosci. 33, 11227-11238. doi: 10.1523/JNEUROSCI.031813.2013

Yamamoto, S., Monosov, I. E., Yasuda, M., and Hikosaka, O. (2012). What and where information in the caudate tail guides saccades to visual objects. J. Neurosci. 32, 11005-11016. doi: 10.1523/JNEUROSCI.082812.2012

Yasuda, M., and Hikosaka, O. (2013). "Functional territories in the primate substantia nigra separately signaling flexible and stable values," in Society for Neuroscience, San Diego, CA.

Yasuda, M., Yamamoto, S., and Hikosaka, O. (2012). Robust representation of stable object values in the oculomotor basal ganglia. J. Neurosci. 32, 16917-16932. doi: 10.1523/JNEUROSCI.3438-12.2012

Yin, H. H., and Knowlton, B. J. (2006). The role of the basal ganglia in habit formation. Nat. Rev. Neurosci. 7, 464-476. doi: 10.1038/nrn1919

Conflict of Interest Statement: The authors declare that the research was conducted in the absence of any commercial or financial relationships that could be construed as a potential conflict of interest.

Received: 04 September 2014; accepted: 10 October 2014; published online: 30 October 2014.

Citation: Kim HF, Ghazizadeh A and Hikosaka O (2014) Separate groups of dopamine neurons innervate caudate head and tail encoding flexible and stable value memories. Front. Neuroanat. 8:120. doi: 10.3389/fnana.2014.00120

This article was submitted to the journal Frontiers in Neuroanatomy.

Copyright (C) $2014 \mathrm{Kim}$, Ghazizadeh and Hikosaka. This is an open-access article distributed under the terms of the Creative Commons Attribution License (CC BY). The use, distribution or reproduction in other forums is permitted, provided the original author(s) or licensor are credited and that the original publication in this journal is cited, in accordance with accepted academic practice. No use, distribution or reproduction is permitted which does not comply with these terms. 